\title{
Ultrahigh-content nitrogen-decorated nanoporous carbon derived from metal organic frameworks and its application in supercapacitors
}

\author{
J. Zou, P. Liu, L. Huang, Q. Zhang, T. Lan, S. Zeng, X. Zeng, L. Yu, S. Liu,
}

H. Wu, W. Tu, Y. Yao

\begin{abstract}
Single electric double-layer capacitors cannot meet the growing demand for energy due to their insufficient energy density. Generally speaking, the supercapacitors introduced with pseudocapacitance by doping heteroatoms $(\mathrm{N}, \mathrm{O})$ in porous carbon materials can obtain much higher capacitance than electric double-layer capacitors. In view of above merits, in this study, nanoporous carbon materials with ultrahigh $\mathrm{N}$ enrichment $(14.23 \mathrm{wt} \%)$ and high specific surface area $\left(942 \mathrm{~m}^{2} \mathrm{~g}^{-1}\right)$ by in situ introduction of N-doped MOF (ZTIF-1, Organic ligands 5methyltetrazole $/ \mathrm{C}_{2} \mathrm{H}_{4} \mathrm{~N}_{4}$ ) were produced. It was found that as supercapacitors' electrode materials, these nanoporous carbons exhibit a capacitance as high as $272 \mathrm{~F} \mathrm{~g}^{-1}$ at $0.1 \mathrm{~A} \mathrm{~g}^{-1}$, and an excellent cycle life (almost no attenuation after 10,000 cycles.). Moreover, the symmetric supercapacitors were assembled to further investigate the actual capacitive performance, and the capacitance shows up to $154 \mathrm{~F} \mathrm{~g}^{-1}$ at $0.1 \mathrm{~A} \mathrm{~g}^{-1}$. Such excellent properties may be attributed to a combination of a high specific surface area, ultrahigh nitrogen content and hierarchically porous structure. The results shown in this study fully demonstrate that the nanoporous carbon materials containing ultrahigh nitrogen content can be used as a potential electrode material in supercapacitors.
\end{abstract}

\section{Keywords}

Ultrahigh nitrogen-decorated, Supercapacitor, MOF-Derived 


\section{Introduction}

Supercapacitor devices, also known as electric double-layer capacitors (EDLCs), store charges by the adsorption of electrolyte ions onto the surface of electrode materials. Compared to traditional batteries, supercapacitors have higher power density and longer cycle life. However, the traditional carbon-based EDLCs show a low capacitance due to the confine of specific surface area (SSA), a single pore structure as well as a bad conductivity on the electron desorption-adsorption surface [1]. Thus, fabricating carbon materials with high SSA, hierarchically porous and good conductivity has become the key for improving the supercapacitor capacitance.

Nanoporous carbon materials derived from metal-organic frameworks (MOFs) that contain metal ions and organic ligands have abundant nanoscale cavities $[2,3]$ that can provide high surfaces for electron desorption-adsorption. Such nanoporous carbon materials have been used as electrode materials for supercapacitors [[4], [5], [6]]. Liu et al. first reported a nanoporous carbon materials (SSA up to $2872 \mathrm{~m} 2 \mathrm{~g}-1$ ) prepared from MOF (PFA/MOF-5 composite) [7]. The electrode materials show a high capacitance up to $258 \mathrm{Fg}-1$ at a current density of 0.25 A g-1. By using ZIF-8-derived nanoporous carbon electrodes, Chaikittisilp et al. obtained a high-capacitance $(214 \mathrm{Fg}-1$ at $5 \mathrm{mV} \mathrm{s}-1)$ using a $0.5 \mathrm{M} \mathrm{H}_{2} \mathrm{SO}_{4}$ as the electrolyte [8]. Moreover, $\mathrm{Hu}$ et al. obtained a nanoporous carbon material with ultrahigh SSA $(5500 \mathrm{~m} 2 \mathrm{~g}-1)$ by direct carbonization of Al-PCP at $800^{\circ} \mathrm{C}$ [9]. Lately, the MOF-derived nanoporous carbon was also reported in energy storage [[10], [11], [12], [13]]. Although commonly nanoporous carbon materials derived from MOFs show a good performance, they still cannot meet the demand for energy storage.

In recent years, nanoporous carbon materials with pseudocapacitive behavior have been reported [[14], [15], [16]]. The introduction of heteroatoms, such as nitrogen $(\mathrm{N})$, phosphorus (P) and oxygen (O), into carbon materials can effectively improve the specific capacitance, 
because the $\mathrm{N}, \mathrm{P}$, O-functional groups can induce pseudocapacitive effects or improve the wettability of carbon materials to electrolyte solution [[17], [18], [19]], especially the introduction of $\mathrm{N}$ element. Moreover, $\mathrm{N}$ doping can improve the electronic conductivity of nanoporous carbon materials $[14,20]$. Thus, the capacitance of nanoporous carbon materials can be enhanced by heteroatom doping.

$\mathrm{N}$-doped carbon materials were prepared by treating carbon materials with ammonia, amines, or urea during the calcination process, or with the direct carbonization of the N-rich precursor, such as polymers, biomass materials [21]. However, the traditional method could only provide either low $\mathrm{N}$ content or low SSA. Thus, developing an approach to obtain high SSA and N content is highly desired.

In this study, a simple route was used to fabricate nitrogen-decorated nanoporous carbon (NNPC) materials with high SSA (942 m2 g-1), hierarchically porous and ultrahigh $\mathrm{N}$ content $(14.23 \mathrm{wt} \%)$ by carbonizing ZTIF-1. Furthermore, the NNPC material was used as electrode materials for supercapacitors and shows an excellent electrochemical performance. A capacitance as high as $272 \mathrm{Fg}-1$ at $0.1 \mathrm{~A} \mathrm{~g}-1$ was obtained when the precursor was calcined at $800{ }^{\circ} \mathrm{C}$ based on the measurement of a 3-electrode configuration. Moreover, the symmetric supercapacitor was assembled to further investigate the actual capacitive performance, and the capacitance shows up to $154 \mathrm{Fg}-1$ at $0.1 \mathrm{Ag}-1$. These results indicate that NNPC materials can be used in supercapacitors for energy storage.

\section{Experimental}

\subsection{Materials}

Zinc acetate $(\mathrm{Zn}(\mathrm{CH} 3 \mathrm{CO} 2) 2)$, ethanol and 5-methyltetrazole were purchased from Macklin. N,N'-dime-thylformide (DMF) was purchased from Sigma Aldrich. 2-ethylimidazole was purchased from TCI. The metal organic framework (ZTIF-1) was prepared by modifying the method reported in Ref. [22]. The mixture of $\mathrm{Zn}(\mathrm{CH} 3 \mathrm{CO} 2) 2)(10 \mathrm{mmol}, 1.83 \mathrm{~g})$, 5- 
methyltetrazole (5-mtz, $10 \mathrm{mmol}, 0.86 \mathrm{~g}$ ), and 2-ethylimidazole (2-eim, $10 \mathrm{mmol}, 0.96 \mathrm{~g}$ ) in DMF $(40 \mathrm{ml})$ and ethanol $(40 \mathrm{ml})$ was sealed in a $150 \mathrm{ml}$ vial and heated to $120^{\circ} \mathrm{C}$ for $72 \mathrm{~h}$, and then cooled to room temperature. The yellow polyhedral crystals (ZTIF-1) were obtained, washed with ethanol, and dried at room temperature.

\subsection{The synthesis of NNPC}

Firstly, the dried ZTIF-1 was carbonized under Ar flow at $700{ }^{\circ} \mathrm{C}, 800^{\circ} \mathrm{C}$ and $900{ }^{\circ} \mathrm{C}$ for $2 \mathrm{~h}$ with a rate of $10^{\circ} \mathrm{C} \mathrm{min}-1$, respectively. Secondly, the NNPC materials were washed by hydrochloric acid to remove the Zn. These samples were named as NNPC-700, NNPC-800 and NNPC-900, respectively.

\subsection{Characterization}

Field emission scanning electron microscopy (FESEM) images were captured by a Hitachi SU70 FESEM instrument, and transmission electron microscopy (TEM) was conducted via a FEI Tecnai G2 F30 facility. Elemental analysis was acquired using a Vario EL cube elemental analyzer. X-ray diffraction (XRD) patterns were recorded on a Bruker D8 advance powder Xray diffractometer using $\mathrm{Cu} \mathrm{K} \alpha$ radiation. The specific surface areas were determined by the gas sorption technique using a Micromeritics ASAP 2020 based on the Brunauere-EmmetteTeller (BET) method. Laser Raman spectroscopy was performed on a Renishawin Via

Spectrometer. X-ray photoelectron spectroscopy (XPS) was investigated on a ULVAC-PHI 1800 spectrometer. The electrochemical properties were examined on an electrochemistry workstation (CHI660E).

\subsection{The fabrication of electrode and electrochemical measurements}

The NNPC electrodes were prepared by mixing $85 \mathrm{wt} \%$ active materials, $10 \mathrm{wt} \%$ acetylene black and $5 \mathrm{wt} \%$ polytetrafluoroethylene with ethanol to form a slurry that was then spread onto a nickel foam with a coating area of $1 \mathrm{~cm} 2$. This coated foam was dried at $100{ }^{\circ} \mathrm{C}$ under vacuum $24 \mathrm{~h}$ and then pressed under a pressure of $10 \mathrm{MPa}$ to completely adhere to the electrode 
materials. The loading mass of active materials on the prepared working electrode is approximately $3 \mathrm{mg} \mathrm{cm}-2$.

Electrochemical measurements were carried out in a standard three-electrode electrochemical cell equipped with a Pt counter electrode and an $\mathrm{Hg} / \mathrm{HgO}$ reference electrode using $6 \mathrm{M} \mathrm{KOH}$ as electrolyte. $\mathrm{CV}$ measurements were performed over the potential range from 0 to $-1 \mathrm{~V}$ in $6 \mathrm{M} \mathrm{KOH}$, at scan rates of $5,10,25,50$ and $100 \mathrm{mV} \mathrm{s}-1$, respectively. Electrochemical impedance spectroscopy (EIS) measurements were conducted over a frequency range between $100 \mathrm{kHz}$ and $10 \mathrm{mHz}$. In a three-electrode configuration, the $\mathrm{C} 3 \mathrm{E}$ was calculated from the discharge curve according to Equation (1):(1)C3E $=\mathrm{I} \Delta \mathrm{TM} \Delta \mathrm{V}$ where $\mathrm{C} 3 \mathrm{E}(\mathrm{F} \mathrm{g}-1), \mathrm{m}(\mathrm{g}), \mathrm{I}(\mathrm{A}$ $\mathrm{g}-1), \Delta \mathrm{t}(\mathrm{s})$, and $\Delta \mathrm{V}(\mathrm{V})$ are specific capacitance, mass of the working electrode, discharge current, discharge time, and potential window of GCD curve, respectively.

Two electrodes were used for the test of elelcrochemical performance in a 2-electrode symmetric supercapacitor configuration. In this configuration, the C2E of NNPC was calculated using Equation (2):(2)C2E $=4 \mathrm{I} \Delta \mathrm{TM} \Delta \mathrm{V}$ where $\mathrm{m}$ is the total mass of the active materials in a device.

The energy density (E) and power density (P) were calculated using Equations (3), (4):(3) $\mathrm{E}=13.6 \times 8 \mathrm{CV} 2$ (4) $\mathrm{P}=\mathrm{E} \Delta \mathrm{T}$ where $\mathrm{C}(\mathrm{F} \mathrm{g}-1)$ represents the specific capacitance of the supercapacitors measured from Equation (2), V (V) refers to the potential change within the discharge time $\Delta \mathrm{T}(\mathrm{s}), \mathrm{E}(\mathrm{Wh} \mathrm{kg}-1)$ is the energy density, and $\mathrm{P}(\mathrm{W} \mathrm{kg}-1)$ is the power density.

\section{Results and discussion}

Scheme 1 illustrates the strategy for the synthesis of NNPC derived from the ultrahigh $\mathrm{N}$ content ZTIF-1, followed by acid wash process. The ZTIF-1 was synthesized by a selfassembly process in a hydrothermal system. In particular, the merit of this approach is that 2ethylimidazole can act as the structure-directing agents and co-linkers to construct a zeolitetype host framework with high porosity and SSA. The introduction of 5-methyltetrazole not 
only plays a key role in construction of the framework. More importantly, it can provide a high in situ N content as a precursor. After pyrolysis and acid wash process, the NNPC was obtained.
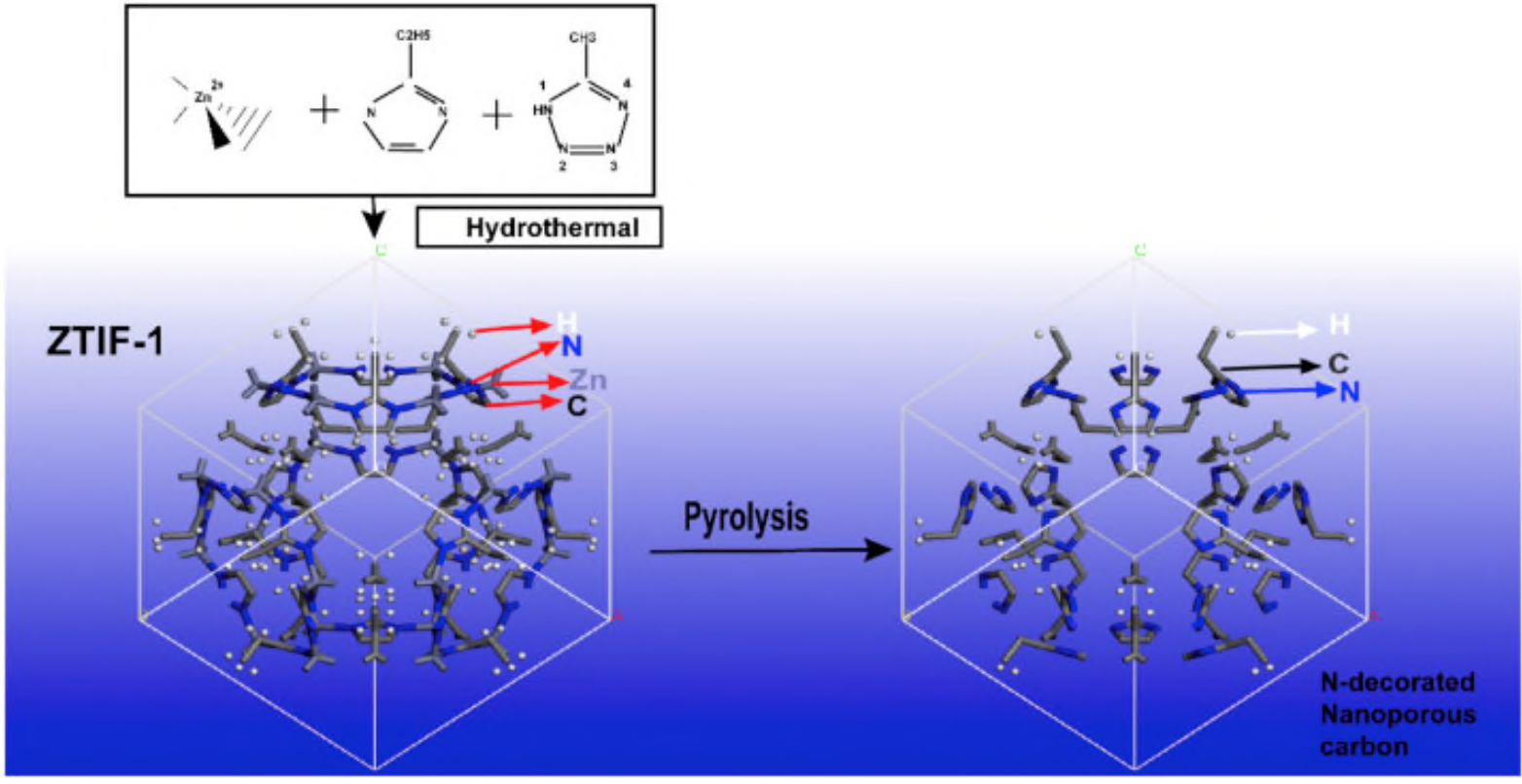

Scheme 1. Schematic illustration for the preparation of NNPC.

Fig. 1a shows the scanning electron microscopy (SEM) image of ZTIF-1, which exhibits threedimensional polyhedral crystals. Moreover, the inset digital photo shows that the crystal is yellow. Furthermore, this synthesized crystal was proved to be ZTIF-1 (CCDC; 1011383) as the literature reported [22]. The XRD pattern of ZTIF-1 was totally corresponding to the simulated structure [22], as shown in Supplementary Fig. S1. Fig. 1b shows a SEM micrograph, highlighting the 3D block-like structure of sample NNPC-800. The hierarchically porous structure of NNPC-800 was clearly observed as shown in Fig. 1c. Supplementary Fig. S2 shows the SEM micrographs of NNPC-700 and 900, and also porous structures could be obtained at the different calcination temperatures. Fig. 1d shows a transmission electron microscopy (TEM) micrograph of NNPC-800, which further proves the porous structure in which consists of micropores and mesopores. Fig. 1e shows a much higher resolution TEM micrograph of NNPC-800, which further highlights a porous structure. 

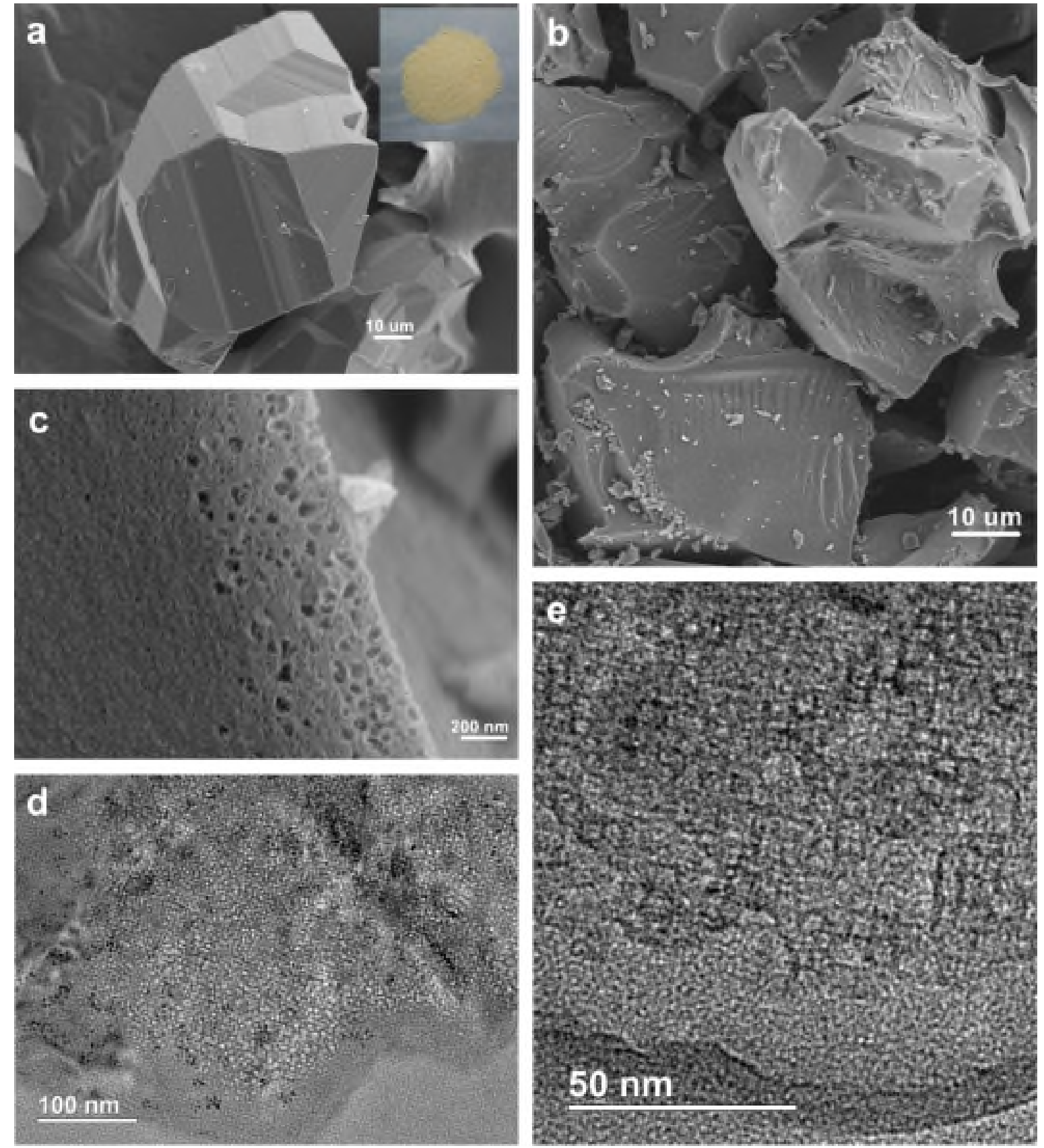

Fig. 1. (a) SEM image and digital image inset of ZTIF-1. SEM (b-c) and TEM (d-e) images of NNPC-800.

The XRD patterns of NNPC-700, 800, and 900 samples are shown in Fig. 2a. It indicates that all the samples have no visible crystalline features although the pyrolysis temperatures for the samples were increased from 700 to $900{ }^{\circ} \mathrm{C}$. The two small signals in the XRD patterns of NNPC at approximately $24^{\circ}, 44^{\circ}(2 \theta)$ can be ascribed to the (002) and (100) diffractions of carbon, respectively. In addition, the (002) peak intensities were drastically decreased with the 
calcination temperature increasing, showing the lower degree of graphitization and the formation of carbon with more defects $[23,24]$. Fig. $2 b$ shows the Raman spectra for different NNPC samples. The G band is located in $\sim 1590 \mathrm{~cm}-1$, corresponding to characteristic features of the graphitic layers. The D band is located in $\sim 1340 \mathrm{~cm}^{-1}$, which relates to disordered carbon or defective graphitic structures. The intensity ratio (ID/IG) of these two peaks depends on the graphitization degree [16,25]. With the ID/IG increasing, the graphitization degree of carbon decreases. The specific ratios of ID/IG for NNPC-700, 800, and 900 samples were 0.87 , 0.98 and 1.02, respectively. Thus, it can be found that the graphitization degree of different NNPC samples decreased when the calcination temperature increased from 700 to $900{ }^{\circ} \mathrm{C}$. This is due to the presence of a large number of defective carbon structures [23]. The SSA and detailed pore structure of the different NNPC samples were analyzed using N2 adsorptiondesorption method, as shown in Fig. 2c-d. As shown in Fig. 2c, the N2 adsorption-desorption isotherm of all NNPC shows a typical type-IV curves with the hysteresis effect and the range is from 0.4 to 0.9. As shown in Supplementary Table S1, the specific SSA of NNPC was calculated based on the BET method, and were 736, 942 and $1018 \mathrm{~m} 2 \mathrm{~g}-1$, respectively. Moreover, the pore size distribution of different NNPC samples was shown in Fig. 2d. All the samples illustrate a hierarchical (micropores/mesopores) structure, which is the result of hysteresis effect. Actually, the large SSA can provide enough surfaces for an electron adsorption-desorption in supercapacitors. Moreover, the hierarchical structure of NNPC can efficiently enlarge the effective utilization rate of SSA because the mesopores can provide quickly ion diffusion pathway in the process of electron adsorption-desorption. 

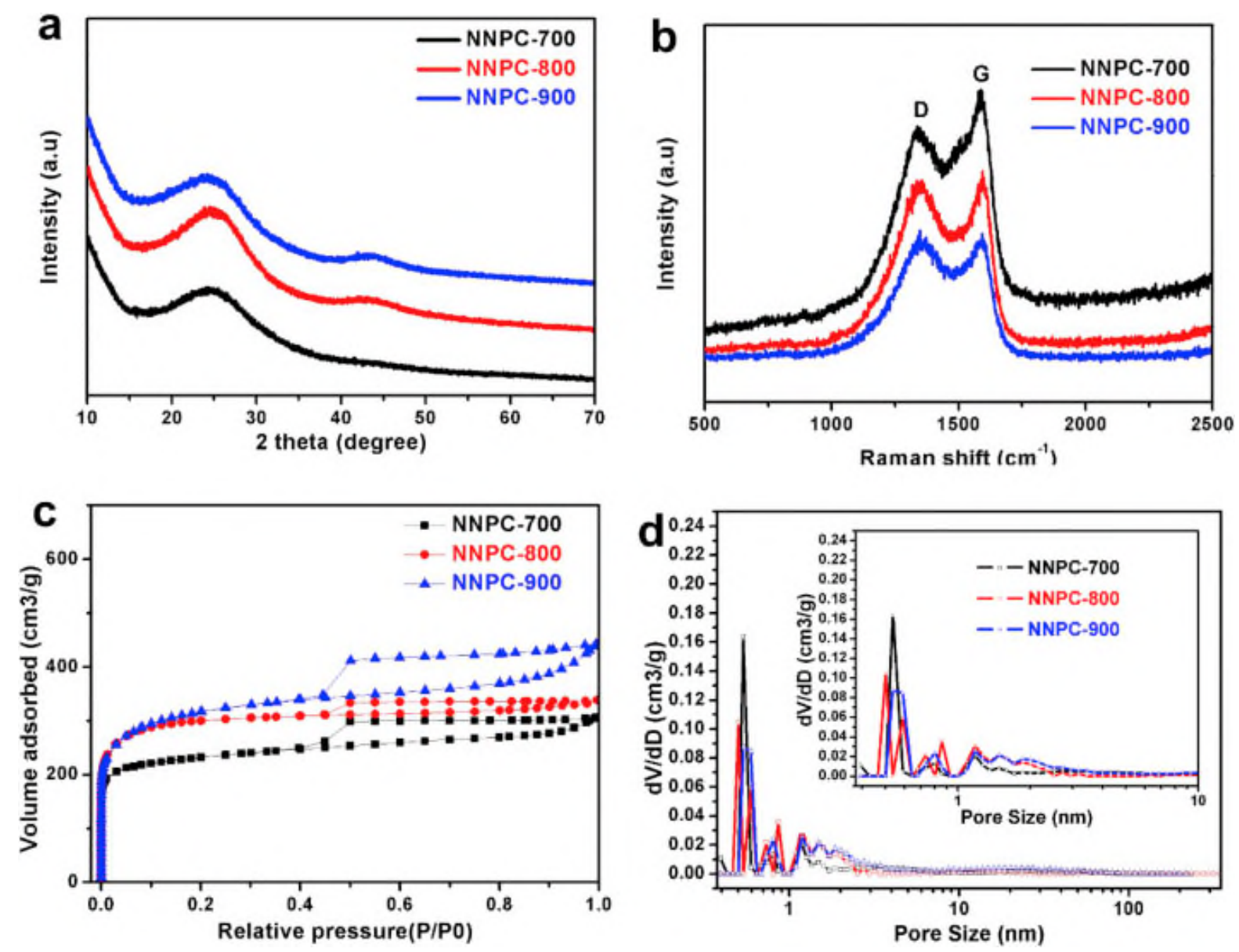

Fig. 2. (a) XRD patterns, (b) Raman spectra, (c) Nitrogen adsorption-desorption isotherms and (d) Pore size distributions of NNPC-700, NNPC-800 and NNPC-900, respectively.

The components in various NNPC samples were analyzed by XPS (Fig. 3). As shown in Fig. 3a-c, the three elements can be clearly observed in NNPC-700, 800 and 900, including carbon, oxygen and nitrogen. Moreover, the EDS results (Supplementary Fig. S3) show that N element is evenly distributed in NNPC-800 sample. For a comparison, the N contents of NNPC-800, and NNPC-900 samples (Supplementary Table S1) was largely decreased to $14.23 \mathrm{wt} \%$ and $6.73 \mathrm{wt} \%$ while the pyrolysis temperature increases. Each high-resolution $\mathrm{O} 1 \mathrm{~s}$ XPS spectrum can be deconvoluted into 3 peaks (Fig. $3 \mathrm{~d}-\mathrm{f}$ ) namely, $=\mathrm{C}=\mathrm{O} /-\mathrm{C}-\mathrm{O}-\mathrm{C}-$ $(\sim 531.0 \pm 0.2 \mathrm{eV}), \quad-\mathrm{O}-\mathrm{C}=\mathrm{O}(\sim 532.0 \pm 0.2 \mathrm{eV}),-\mathrm{N}-\mathrm{O}-(\sim 534.0 \pm 0.2 \mathrm{eV}) . \quad$ In particular, the portion change of $\mathrm{N}$-oxide is not obvious when increasing the calcination temperature from $800{ }^{\circ} \mathrm{C}$ to $900{ }^{\circ} \mathrm{C}$, which illustrates the stability of N-oxide in NNPC. The hydrophilic groups 
could drastically improve the wettability between electrolyte ions and the electrode active materials, including carboxyl (-COOH), epoxyl (C-O-C) and oxidized $\mathrm{N}$ (Fig. 3d-f). The high-resolution XPS spectra of N1s were shown in Fig. 2 g-i, and can be deconvoluted into 4 peaks, representing pyridinic $\mathrm{N}(\mathrm{N}-6$ at $398.0 \pm 0.2 \mathrm{eV})$, pyrrolic $\mathrm{N}(\mathrm{N}-5$ at $400.10 .2 \pm \mathrm{eV})$, quaternary $\mathrm{N}(\mathrm{N}-\mathrm{Q}$ at $400.9 \pm 0.2 \mathrm{eV})$ and oxidized $\mathrm{N}(\mathrm{N}-\mathrm{X}$ at $404.0 \pm 0.2 \mathrm{eV})$. Moreover, the proportion of quaternary $\mathrm{N}$ was drastically increased and the pyridinic $\mathrm{N}$ and pyrrolic $\mathrm{N}$ were decreased with the calcination temperature rising. As illustrated in the literature, the N-5 and N-6 play a crucial role to contributing to pseudocapacitance and improve the wettability of materials [[26], [27], [28]]. Thus, too high calcination temperature is not beneficial to obtaining the high capacity because of the decreases of N-6 and N-5.
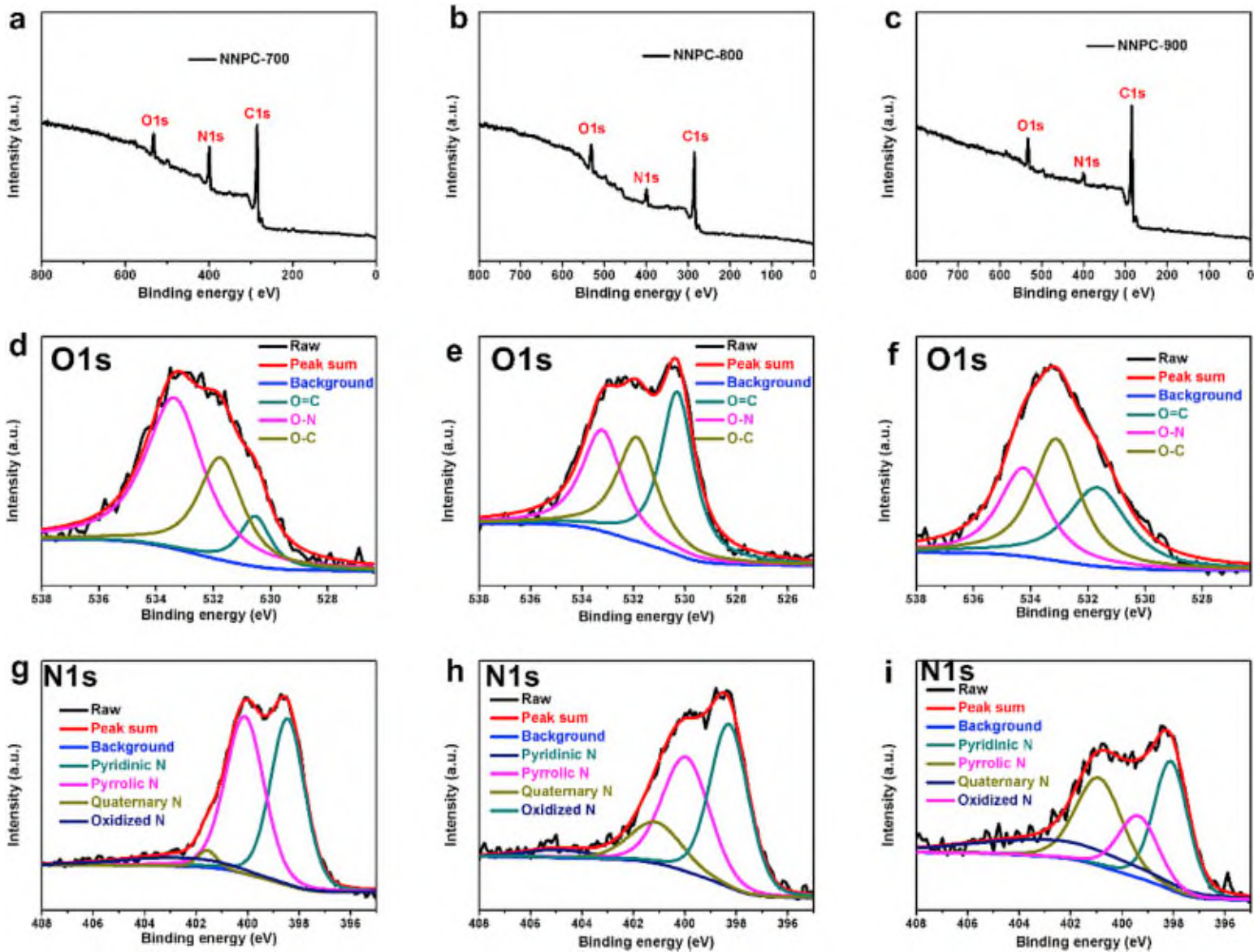

Fig. 3. XPS results of the NNPC-700, NNPC-800 and NNPC-900 samples. (a-c) XPS survey, (d-f) O1s spectra, (g-i) N1s spectra. The left, middle and right columns are the results from the NNPC-700, NNPC-800 and NNPC-900 samples, respectively. 
To investigate the electrochemical performance of as-prepared different NNPC materials used as electrodes for supercapacitors, the electrochemical measurements were carefully carried out in $6 \mathrm{M} \mathrm{KOH}$ aqueous electrolyte with a three-electrode configuration. Fig. 4a shows the CV curves of different NNPC materials within a potential window of -1 to $0 \mathrm{~V}$ at a scan rate of $10 \mathrm{mV} \mathrm{s}-1$. The NNPC-800 and NNPC-900 electrodes show similar CV profiles with a slightly twisted rectangular shape. However, the NNPC-700 exhibits irregular CV curves, resulting from the poor conductivity. The Nyquist plots of NNPC-700, 800 and 900 were shown in Supplementary Fig. S4 and further proved the bad conductivity of NNPC-700 (the largest semicircle exhibits the large charge transfer resistance). As shown in Fig. 4b, the GCD curves of NNPC-800 exhibit the longest discharge time in all samples and highly linear and symmetrical, demonstrating an excellent electrochemical reversibility. By contrast, the NNPC700 exihibts a non-linear GCD curve, corresponding to CV curve analysis. Significantly, the gravimetric specific capacitances of NNPC-700, 800 and 900 at the current densities of $0.1 \mathrm{Ag}-1$ were calculated based on equation (1) and the values are 158, 272 and $225 \mathrm{~F} \mathrm{~g}-1$, respectively (Fig. 4c). When the current densities up to $10 \mathrm{Ag}-1$, the specific capacitance retention of NNPC-700, 800 and 900 is $9.36 \%, 54.4 \%$ and 52\%, respectively. To further understand the better electrochemical performance for NNPC-800, CV curves at different scan rates were plotted and shown in Fig. 4d. All CV curves of NNPC-800 exhibit rectangular-like shape, even at a high rate of $100 \mathrm{mVs}-1$, indicating excellent capacitive behavior [29]. Moreover, the NNPC-800 illustrates an excellent cycle performance at a current density of $5 \mathrm{Ag}-1$, as shown in Fig. 4e. The specific capacitance of NNPC-800 is as high as $166 \mathrm{~F} \mathrm{~g}-1$, with almost no attenuation after 10,000 cycles. The good electrochemical performance can be ascribed to the excellent conductivity of NNPC-800. In addition, the Nyquist plots of NNPC800 before and after cycle 10,000 times also prove this result. Rct represents the charge-transfer resistance between the electrolyte and electrode. It can be found that the Rct (the semicircle in 
the high frequency) was $1.0 \Omega$ before cycle, and after cycling 10,000 times the Rct only slightly increased to $1.25 \Omega$. Thus, all electrochemical tests so far illustrate the good performance of NNPC-800 in a three-electrode system.
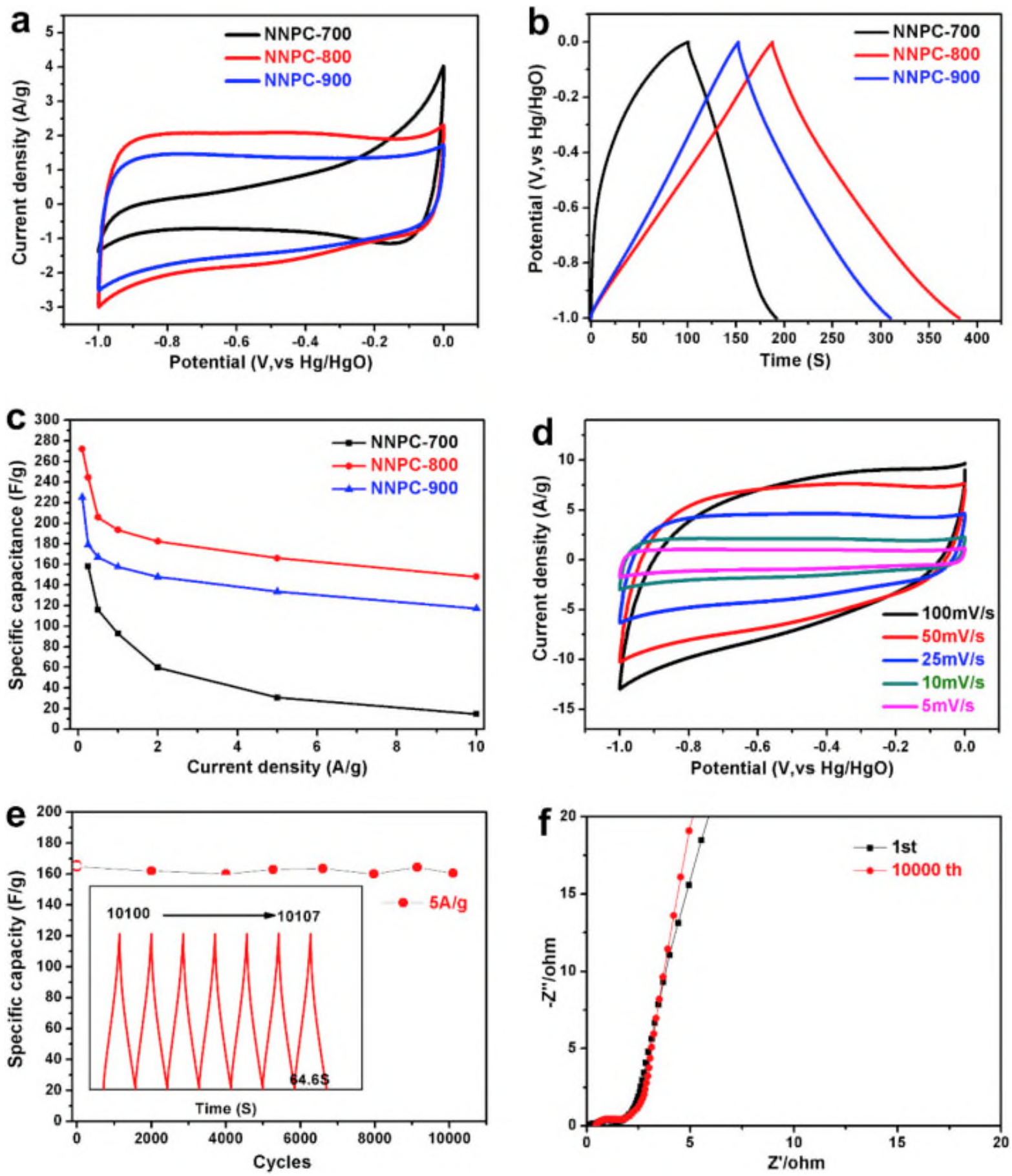

Fig. 4. (a) CV curves at a scan rate of $10 \mathrm{mV} \mathrm{s}-1$ (b) GCD curves at acurrent density of

1 A g-1 and (c) The specific capacityat various current densities of NNPC-700, 800 and 900, 
respectively. (d) CV curves of NNPC-800 at different scan rates from 5 to $100 \mathrm{mV} \mathrm{s}-1$. (e)

Cycle performance at $5 \mathrm{~A} \mathrm{~g}-1$ and (f) Nyquist plots after 10,000 cycles of NNPC-800.

To further evaluate the capacitive performance of the NNPC-800 electrode in a full cell set-up, a symmetric supercapacitor based on a two-electrode system was assembled in $6 \mathrm{M} \mathrm{KOH}$ aqueous electrolyte. Fig. 5a shows the CV curves for NNPC-800. It is clear that CV curves (5$50 \mathrm{mV} \mathrm{s}-1)$ show quasi-rectangular shapes within a cell voltage of $1.0 \mathrm{~V}$, which is characteristic of electrochemical double-layer capacitor. When the scan rate reaches up to $100 \mathrm{mVs}-1$, the CV shape shows an abnormal quasi-rectangular, which may be attributed to the contribution of pseudocapacitance (N-doped) [14,15]. As shown in Fig. 5b, the specific GCD curves illustrate long discharging time, which further proves a high capacitance of NNPC-800 electrode in a two-electrode system. Moreover, the specific capacitance of NNPC800 was calculated based on equation (2) and shown in Fig. 5c. The high capacitance (154 F g-1) was obtained at $0.1 \mathrm{Ag}-1$ current density. After the current density increases to 20 times (2 A g-1), the capacitance retention (57\%) can still maintain. A low internal resistance of $4 \Omega$ suggests good conductivity of NNPC-800 symmetric supercapacitor. The inset of Fig. $5 \mathrm{~d}$ is the equivalent circuit for the fitting of EIS data by Zview software. The simulation curve almost overlaps the original EIS curve, indicating a good fitting. The equivalent circuit consists of the following parts: L, Rs, Rct, W, Cdl, CL [[30], [31], [32]]. L stands for an inductance; Rs is the sum of contact resistance, including the resistance of active materials and the resistance of electrolyte; Rct represents the charge-transfer resistance between the electrolyte and electrode; $\mathrm{W}$ is the Warburg resistance; Cdl and the CL are capacitance of the double-layer and the limit pseudocapacitance stemming from redox reaction of heteroatom containing groups, respectively. 

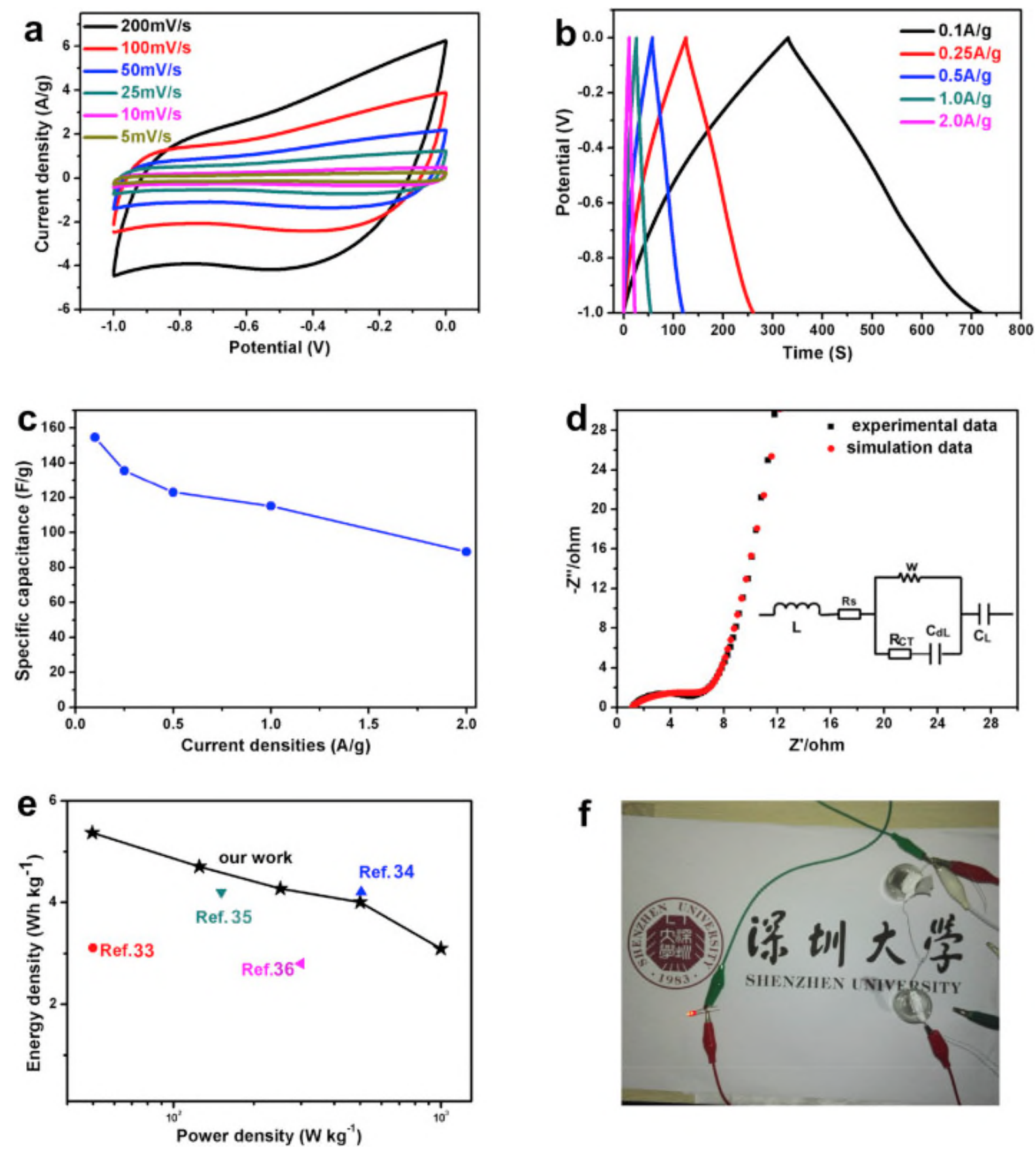

Fig. 5. CV curves (a) and GCD curves (b) for NNPC-800 tested in a two electrode system. (c) The rate performance of NNPC-800 at different current densities in a two-electrode system. (d) Nyquist plots of NNPC-800 in a two-electrode system. (e)The Ragone plots of our symmetric supercapacitor and other previously reported. (f)The digital image of LED with light up by a device of two supercapacitors connected in series.

As shown in Fig. 5e, the power density and energy density of NNPC-800 were obtained based on equations (3), (4), and the energy density of $5.36 \mathrm{Wh} \mathrm{kg-1}$ were obtained when the power 
density is $49.7 \mathrm{~W} \mathrm{~kg}-1$ in the assembled symmetric supercapacitors, which shows better results than previously reported nanoporous carbon symmetric supercapacitors in aqueous electrolytes [[33], [34], [35], [36]]. An energy storage device with two supercapacitors connected in series was fabricated. Such a simple energy storage device could power a commercial red lightemitting diode (LED) as shown in Fig. 5f. Thus, it has further proved the potential commercial value of NNPC materials in energy storage.

Therefore, the superior electrochemical performance of NNPC electrode can be attributed to the synergistic effect of its hierarchically pore structure with high SSA and N,O heteroatom doping. On the one hand, as shown in Fig. 6a macropores can store a lot of electrolyte, and mesopores can provide channels for ions approaching the carbons surface and micropores can provide a large number of adsorption-desorption sites for electrons to transport, all of which facilitate the fast transportation of ions and electron [1,[37], [38], [39], [40], [41]]. On the other hand, the introduction of N,O heteroatom leads to functionalization of NNPC surface (Fig. 6b). Some special functional group can improve the wettability of electrode surface and electrolyte, such as $-\mathrm{OH}, \mathrm{C}=\mathrm{O}$, especially in $\mathrm{N}$-doped carbon $[26,27,42]$. Moreover, as some literature demonstrated that the NNPC (pyrrolic $\mathrm{N}$ and pyridinic $\mathrm{N}$ ) materials can obtain the extra pseudocapacitance [18,[43], [44], [45]]. Thus, hierarchical pore structure with high SSA and $\mathrm{N}$-enrichment contributes to the high capacitance and excellent rate performance.

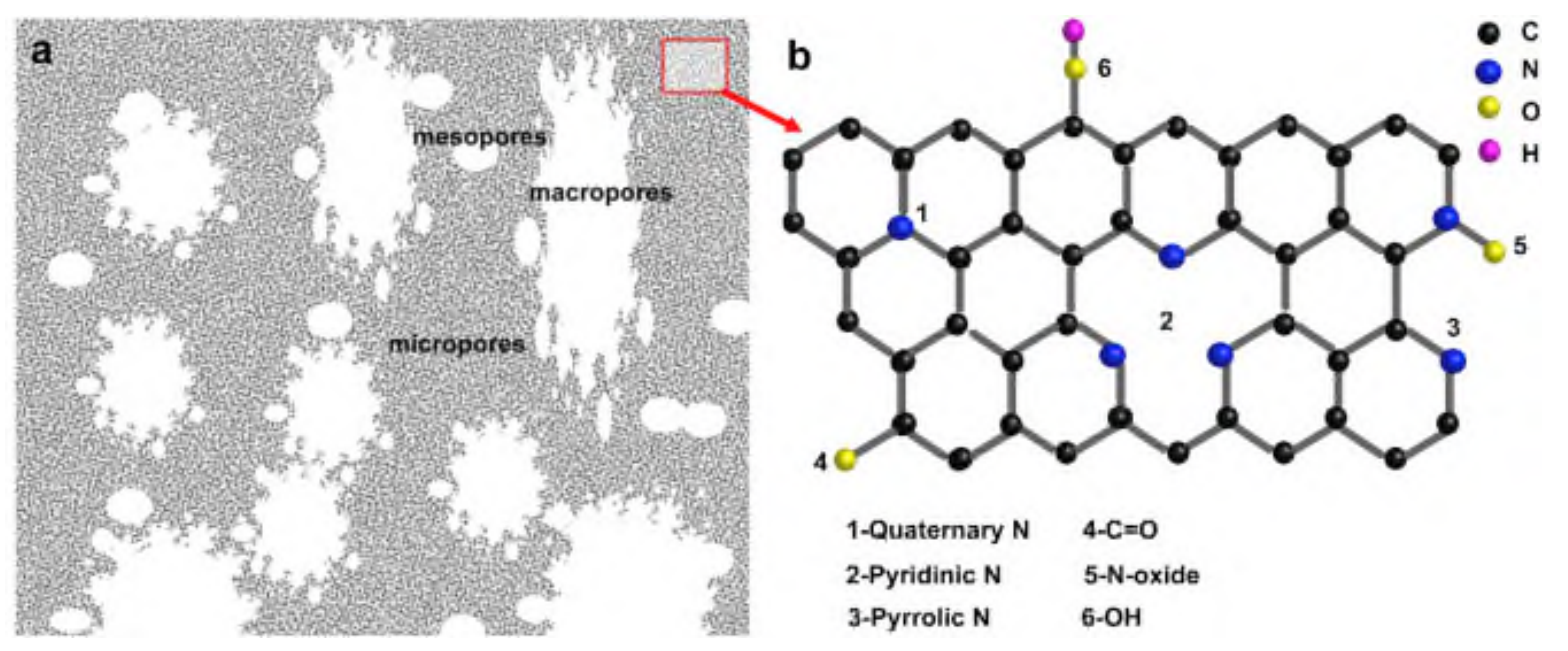


Fig. 6. (a) The schematic of hierarchical pore structure for NNPC materials. (b) The illustration of functionality group for NNPC materials.

\section{Conclusions}

In summary, ultrahigh nitrogen-decorated nanoporous carbon was prepared through calcination of the ultrahigh nitrogen MOF by hydrothermal synthesis. In this study, the obtained porous carbon material with its high surface area $(942 \mathrm{~m} 2 \mathrm{~g}-1)$, hierarchical pore structure, and ultrahigh nitrogen-doping (14.23 wt\%) shows a high specific gravimetric capacitance of $272 \mathrm{~F} \mathrm{~g}-1$, and an excellent cycling stability of $100 \%$ capacitance retention after 10,000 cycles. More importantly, the assembled symmetric supercapacitors deliver a high specific gravimetric capacitance of $154 \mathrm{~F} \mathrm{~g}-1$ at $0.1 \mathrm{Ag}-1$ and a superb gravimetric energy density of $5.36 \mathrm{Wh} \mathrm{kg}-1$ when the power density is $49.7 \mathrm{~W} \mathrm{~kg}-1$. These results illustrate the prepared NNPC electrode material may be a promising candidate for high performance supercapacitors.

Acknowledgments

This work was financially supported by the National Natural Science Foundation of China (Nos.51202150, 51272161,21703141), Program of Introducing Innovative Research Team in Dongguan (No.2014607109), foundation of the State Key Laboratory of Solidifi-cation Processing in NWPU(SKLSP201110), Shenzhen Basic Research Progr-am (No. JCYJ20160422091418366) and foundation of Shenzhen University Innovation and Development (PIDFP-ZR2017022).

\section{References}

[1] C. Merlet, B. Rotenberg, P.A. Madden, P.L. Taberna, P. Simon, Y. Gogotsi, M. Salanne Nat. Mater., 11 (2012), pp. 306-310

[2] J. Tang, Y. Yamauchi Nat. Chem., 8 (2016), pp. 638-639 
[3] R.R. Salunkhe, Y.V. Kaneti, J. Kim, J.H. Kim, Y. Yamauchi Acc. Chem. Res., 49 (2016), pp. 2796-2806

[4] C. Qu, Y. Jiao, B. Zhao, D. Chen, R. Zou, K.S. Walton, M. Liu Nanomater. Energy, 26 (2016), pp. 66-73

[5] H. Wang, Q.-L. Zhu, R. Zou, Q. Xu Inside Chem., 2 (2017), pp. 52-80

[6] C. Qu, B. Zhao, Y. Jiao, D. Chen, S. Dai, B.M. deglee, Y. Chen, K.S. Walton, R. Zou, M. Liu

ACS Energy Letters, 2 (2017), pp. 1263-1269

[7] B. Liu, H. Shioyama, T. Akita, Q. Xu J. Am. Chem. Soc., 130 (2008), p. 5390

[8] W. Chaikittisilp, M. Hu, H. Wang, H.S. Huang, T. Fujita, K.C. Wu, L.C. Chen, Y. Yamauchi, K. Ariga

Chem Commun (Camb), 48 (2012), pp. 7259-7261

[9] M. Hu, J. Reboul, S. Furukawa, N.L. Torad, Q. Ji, P. Srinivasu, K. Ariga, S. Kitagawa, Y. Yamauchi

J. Am. Chem. Soc., 134 (2012), pp. 2864-2867

[10] R.R. Salunkhe, C. Young, J. Tang, T. Takei, Y. Ide, N. Kobayashi, Y. Yamauchi Chem Commun (Camb), 52 (2016), pp. 4764-4767

[11] W. Xia, C. Qu, Z. Liang, B. Zhao, S. Dai, B. Qiu, Y. Jiao, Q. Zhang, X. Huang, W. Guo, D. Dang, R. Zou, D. Xia, Q. Xu, M. Liu, Nano Lett., 17 (2017), pp. 2788-2795

[12] P. Pachfule, D. Shinde, M. Majumder, Q. Xu Nat. Chem., 8 (2016), pp. 718-724

[13] S. Dang, Q.-L. Zhu, Q. Xu Nat. Review. Mater., 3 (2017), p. 17075

[14] M. Zhou, F. Pu, Z. Wang, S. Guan Carbon, 68 (2014), pp. 185-194

[15] L. Fan, L. Yang, X. Ni, J. Han, R. Guo, C. Zhang Carbon, 107 (2016), pp. 629-637

[16] Z. Li, Z.W. Xu, X.H. Tan, H.L. Wang, C.M.B. Holt, T. Stephenson, B.C. Olsen, D. Mitlin Energy Environ. Sci., 6 (2013), pp. 871-878 
[17] C. Huang, A.M. Puziy, T. Sun, O.I. Poddubnaya, F. Suárez-García, J.M.D. Tascón, D. Hulicova-Jurcakova Electrochim. Acta, 137 (2014), pp. 219-227

[18] X. Yan, Y. Liu, X. Fan, X. Jia, Y. Yu, X. Yang J. Power Sources, 248 (2014), pp. $745-$ 751

[19] Y. Zhai, Y. Dou, D. Zhao, P.F. Fulvio, R.T. Mayes, S. Dai Adv. Mater., 23 (2011), p. 4828

[20] J.P. Paraknowitsch, A. Thomas, M. Antonietti J. Mater. Chem., 20 (2010)

[21] B. Zhu, B. Liu, C. Qu, H. Zhang, W. Guo, Z. Liang, F. Chen, R. Zou J. Mater. Chem. (2018)

[22] F. Wang, H.R. Fu, Y. Kang, J. Zhang Chem Commun (Camb), 50 (2014), pp. 1206512068

[23] Z. Wen, X. Wang, S. Mao, Z. Bo, H. Kim, S. Cui, G. Lu, X. Feng, J. Chen Adv. Mater., 24 (2012), p. 5610

[24] X. Liu, A. Markus Adv. Mater., 25 (2013), pp. 6284-6290

[25] S.D.M. Brown, A. Jorio, P. Corio, M.S. Dresselhaus, G. Dresselhaus, R. Saito, K. Kneipp Phys. Rev. B, 63 (2001), Article 155414

[26] C. Chen, D.F. Yu, G.Y. Zhao, B.S. Du, W. Tang, L. Sun, Y. Sun, F. Besenbacher, M. Yu Nanomater. Energy, 27 (2016), pp. 377-389

[27] B. Duan, X. Gao, X. Yao, Y. Fang, L. Huang, J. Zhou, L. Zhang, Nanomater. Energy, 27 (2016), pp. 482-491

[28] D. Usachov, O. Vilkov, A. Gruneis, D. Haberer, A. Fedorov, V.K. Adamchuk, A.B. Preobrajenski, P. Dudin, A. Barinov, M. Oehzelt, C. Laubschat, D.V. Vyalikh, Nano Lett., 11 (2011), pp. 5401-5407

[29] C. Long, X. Chen, L. Jiang, L. Zhi, Z. Fan, Nanomater. Energy, 12 (2015), pp. 141-151

[30] Y. Wang, H. Xuan, G. Lin, F. Wang, Z. Chen, X. Dong, J. Power Sources, 319 (2016), pp. $262-270$ 
[31] Z. Tai, X. Yan, J. Lang, Q. Xue, J. Power Sources, 199 (2012), pp. 373-378

[32] S.Y. Kim, K.S. Yang, B.H. Kim Electrochim. Acta, 137 (2014), pp. 781-788

[33] J. Zhang, J. Jiang, H. Li, X.S. Zhao Energy Environ. Sci., 4 (2011), p. 4009

[34] S. Suresh Balaji, M. Karnan, M. Sathish Int. J. Hydrogen Energy (2017)

[35] A. Kumar, A. Sanger, A. Kumar, Y. Kumar, R. Chandra Electrochim. Acta, 222 (2016), pp. 1761-1769

[36] Y. Dong, H.M. Lin, D. Zhou, H. Niu, Q.M. Jin, F.Y. Qu Electrochim. Acta, 159 (2015), pp. $116-123$

[37] W. Bao, A.K. Mondal, J. Xu, C. Wang, D. Su, G. Wang J. Power Sources, 325 (2016), pp. 286-291

[38] Y. Lu, K. Fu, S. Zhang, Y. Li, C. Chen, J. Zhu, M. Yanilmaz, M. Dirican, X. Zhang J. Power Sources, 273 (2015), pp. 502-510

[39] C. Portet, D. Kazachkin, S. Osswald, Y. Gogotsi, E. Borguet, Thermochim. Acta, 497 (2010), pp. 137-142

[40] M. Rose, Small, 7 (2011), pp. 1108-1117

[41] A.J. Amali, J.K. Sun, Q. Xu Chem Commun (Camb), 50 (2014), pp. 1519-1522

[42] X. Jiang, L.X. Sun, F. Xu Materials Science Forum (2016), pp. 829-834

[43] Y.S. Yun, H.H. Park, H.J. Jin, Materials, 5 (2012), pp. 1258-1266

[44] F. Sun, J.H. Gao, X.X. Pi, L.J. Wang, Y.Q. Yang, Z.B. Qu, S.H. Wu, J. Power Sources, 337 (2017), pp. 189-196

[45] Z.-H. Huang, T.-Y. Liu, Y. Song, Y. Li, X.-X. Liu Nanoscale (2017) 
2018-04-03

Ultrahigh-content nitrogen-decorated nanoporous carbon derived from metal organic frameworks and its application in supercapacitors

Zou, Jizhao

Elsevier

Yao Y, Tu W, Zou J, et al., (2018) Ultrahigh-content nitrogen-decorated nanoporous carbon derived from metal organic frameworks and its application in supercapacitors. Electrochimica Acta, Volume 271, May 2018, pp. 599-607

https://doi.org/10.1016/j.electacta.2018.03.200

Downloaded from Cranfield Library Services E-Repository 